\title{
Entropy Solutions of Doubly Nonlinear Fractional Laplace Equations
}

\author{
Niklas Grossekemper, Petra Wittbold, and \\ Aleksandra Zimmermann®
}

\begin{abstract}
In this contribution, we study a class of doubly nonlinear elliptic equations with bounded, merely integrable right-hand side on the whole space $\mathbb{R}^{N}$. The equation is driven by the fractional Laplacian $(-\Delta)^{\frac{s}{2}}$ for $s \in(0,1]$ and a strongly continuous nonlinear perturbation of first order. It is well known that weak solutions are in genreral not unique in this setting. We are able to prove an $L^{1}$-contraction and comparison principle and to show existence and uniqueness of entropy solutions.
\end{abstract}

Mathematics Subject Classification. 35S10, 35S30, 35L67.

Keywords. Fractional Laplacian, strongly continuous perturbation, entropy solution, vanishing viscosity, $L^{1}$-data, doubly nonlinear.

\section{Introduction}

Let $N \in \mathbb{N}$. We consider the doubly nonlinear fractional Laplace equation

$$
b(u)+\operatorname{div}(F(u))+(-\Delta)^{\frac{s}{2}} u=f \quad \text { in } \mathbb{R}^{N}
$$

where $f \in L^{1}\left(\mathbb{R}^{N}\right) \cap L^{\infty}\left(\mathbb{R}^{N}\right)$ and $b: \mathbb{R} \rightarrow \mathbb{R}$ is Lipschitz continuous, nondecreasing with $b(0)=0$, satisfying the growth condition $b(u) u \geq \lambda|u|^{2}$ for some $\lambda>0$. For example, these assumptions are fulfilled by $b=I d+\arctan$ or $b=I d+\sin$, and it is also possible for $b$ to be constant on finite intervals. The function $F: \mathbb{R} \rightarrow \mathbb{R}^{N}$ is locally Lipschitz continuous with $F(0)=0$ and $(-\Delta)^{\frac{s}{2}}$ is the fractional Laplacian with $s \in(0,1]$ (see Sect. 2 for the precise definition).

The fractional Laplacian is a nonlocal generalization of the classical Laplacian which appears in many fields of analysis and probability theory. In the 
last two decades, there has been an intensive study of elliptic and evolutionary partial differential equations driven by the fractional Laplacian or related nonlocal operators, see, e.g., [16] for a list of interesting references. In applications from physics and finance, anomalous diffusion is often modeled by a fractional Laplace evolution equation (see [7], Appendix B for more details and references).

In this contribution, we study doubly nonlinear elliptic equations of type $\left(\mathscr{P}_{f}\right)$ with bounded right-hand side in $L^{1}\left(\mathbb{R}^{N}\right)$ on the whole space $\mathbb{R}^{N}$. The equation is driven by the fractional Laplacian $(-\Delta)^{\frac{s}{2}}$ for $s \in(0,1]$ and a strongly continuous perturbation of first order of the form $\operatorname{div} F$ with $F: \mathbb{R} \rightarrow$ $\mathbb{R}^{N}$ Lipschitz continuous.

In [11] it was shown that adding a fractional Laplacian with parameter $s \in(1,2)$ to a hyperbolic equation has a smoothing effect, i.e., weak solutions exist and are unique. In [2], the fractional Burgers equation was studied for $s \in(0,1)$ and it was shown that weak solutions are not unique. Analogously to the purely hyperbolic case (see [15]), an entropy formulation for fractional scalar conservation laws has been developped in [1]. Consequently, one can not expect well-posedness of weak solutions of $\left(\mathscr{P}_{f}\right)$ even in the case $b(u)=u$ and therefore one has to choose a more appropriate solution concept. The well-posedness of $\left(\mathscr{P}_{f}\right)$ for $F \equiv 0$ has been studied in [3] in the framework of renormalized solutions. However, since $\left(\mathscr{P}_{f}\right)$ can be interpreted as a special case of a fractional Laplace evolution equation with a first-order convection term, it seems to be more natural to apply the notion of entropy solution in our general setting. In [5], entropy solutions have been introduced for elliptic equations with $L^{1}$-data. In this contribution, we define the notion of entropy solutions for $\left(\mathscr{P}_{f}\right)$. Moreover, we show existence and the $L^{1}$-contraction and comparison principles for entropy solutions. In particular, we obtain uniqueness of $b(u)$ in this framework. We recall that existence and $L^{1}$-contraction allows us to define the $m$-accretive, densely defined, multivalued operator $A_{b}$ in $L^{1}\left(\mathbb{R}^{N}\right)$ by

$$
\begin{aligned}
& (v, f) \in A_{b} \Longleftrightarrow \\
& v=b(u), u \in L^{1}\left(\mathbb{R}^{N}\right) \text { is entropy solution to } \operatorname{div}(F(u))+(-\Delta)^{\frac{s}{2}} u=f .
\end{aligned}
$$

According to nonlinear semigroup theory (see, e.g., [4]), there exists a unique mild solution $b \in C\left([0, T] ; L^{1}\left(\mathbb{R}^{N}\right)\right)$ to the abstract Cauchy problem for $A_{b}$ for any given data $\left(v_{0}, f\right) \in L^{1}\left(\mathbb{R}^{N}\right) \times L^{1}\left(0, T ; L^{1}\left(\mathbb{R}^{N}\right)\right)$. In the next step, one wants to show that the mild solution is the unique entropy solution to the associated evolution equation

$$
\begin{aligned}
b(u)_{t}+\operatorname{div}(F(u))+(-\Delta)^{\frac{s}{2}} u & =f \quad \text { in }(0, T) \times \mathbb{R}^{N} \\
u(0, \cdot) & =u_{0}
\end{aligned}
$$

for appropriately chosen data $\left(u_{0}, f\right) \in L^{1}\left(\mathbb{R}^{N}\right) \times L^{1}\left(0, T ; L^{1}\left(\mathbb{R}^{N}\right)\right)$. In this manner, our study of $\left(\mathscr{P}_{f}\right)$ serves as a basis for the investigation of $(1)$, which 
will be subject of forthcoming work. In the special case of an invertible nonlinearity $b$ with Lipschitz continuous inverse, evolution equation (1) with a multiplicative stochastic noise term on the right-hand side has been addressed in $[6]$.

\subsection{Outline}

We organize this contribution as follows: We start in Sect. 2 by introducing some basic notations which we will use throughout the paper and give some preliminary results that are used within the later sections. After that, in Sect. 3, we establish the definition of an entropy solution to the equation $\left(\mathscr{P}_{f}\right)$ and formulate the two main theorems of this work. In Sect. 4 we prove the $L^{1}$ contraction and comparison principle with the help of Kruzhkov's method of doubling variables. Since this result is also crucial for the existence proof later on, it is proven before the existence of entropy solutions. Furthermore, the contraction principle gives us uniqueness of the saturation function $b(u)$ and in some cases even the uniqueness of the entropy solution $u$ itself. Finally, in Sect. 5 we prove the existence of entropy solutions. For this, we apply the method of vanishing viscosity to be able to show that there exist weak solutions for a sequence of approximating problems of higher regularity. It is then left to show that these weak solutions converge to the entropy solution of the initial problem. In the "Appendix", for the sake of completeness, some of the technical results used in this work are proven.

\section{Notations and Preliminary Results}

Let us introduce some notations and functions that will be frequently used. For any real number $r \in \mathbb{R}$ and $k>0$, we define the sign functions

$$
\operatorname{sign}_{0}(r)=\left\{\begin{array}{ll}
1 & r>0 \\
0 & r=0 \\
-1 & r<0
\end{array}, \quad \operatorname{sign}_{0}^{+}(r)= \begin{cases}1 & r>0 \\
0 & r \leq 0\end{cases}\right.
$$

as well as the truncation function

$$
T_{k}(r)=\left\{\begin{array}{ll}
k & r>k \\
r & |r| \leq k \\
-k & r<-k
\end{array} .\right.
$$

For all $u \in \mathscr{S}\left(\mathbb{R}^{N}\right)$, the Schwartz space of rapidly descreasing functions, and all $s \in(0,2)$, we define the fractional Laplacian $(-\Delta)^{\frac{s}{2}}$ by

$$
(-\Delta)^{\frac{s}{2}} u(x)=C(N, s) P . V . \int_{\mathbb{R}^{N}} \frac{u(x)-u(y)}{|x-y|^{N+s}} \mathrm{~d} y
$$




$$
=C(N, s) \lim _{\varepsilon \rightarrow 0} \int_{\mathbb{R}^{N} \backslash B_{\varepsilon}(x)} \frac{u(x)-u(y)}{|x-y|^{N+s}} \mathrm{~d} y,
$$

where the dimensional constant $C(N, s)>0$ is given by $C(N, s)=\frac{s \Gamma\left(\frac{N+s}{2}\right)}{2 \pi^{\frac{N}{2}+s} \Gamma\left(1-\frac{s}{2}\right)}$ with $\Gamma$ being the gamma function. The constant $C(N, s)$ is motivated by an equivalent definition of the fractional Laplacian via Fourier transform, i.e., by $(-\Delta)^{\frac{s}{2}} u=\mathscr{F}^{-1}\left(|\cdot|^{s} \mathscr{F}(u)\right)$, where it naturally occurs. We further define the Gagliardo-seminorm by

$$
[u]_{s / 2}:=\left(\int_{\mathbb{R}^{N}} \int_{\mathbb{R}^{N}} \frac{|u(x)-u(y)|^{2}}{|x-y|^{N+s}} \mathrm{~d} x \mathrm{~d} y\right)^{\frac{1}{2}}
$$

and the fractional Sobolev space of order $\frac{s}{2}$ by

$$
H^{\frac{s}{2}}\left(\mathbb{R}^{N}\right):=\left\{u \in L^{2}\left(\mathbb{R}^{N}\right):[u]_{s / 2}<\infty\right\} .
$$

As it is well-known, this fractional Sobolev space is a Hilbert space, if endowed with the natural scalar product which induces the norm

$$
\|u\|_{s / 2}=\left(\|u\|_{L^{2}}^{2}+[u]_{s / 2}^{2}\right)^{\frac{1}{2}} .
$$

We point out that the fractional Sobolev space $H^{\frac{s}{2}}\left(\mathbb{R}^{N}\right)$ is strictly related to the fractional Laplacian (see [10] Proposition 3.6).

An important tool will be a decomposition of the fractional Laplacian which was introduced by Droniou and Imbert in [12] Theorem 1, where the authors split the fractional Laplacian into a regular and a singular part.

Proposition 1. If $s \in(0,2)$, then for all $u \in \mathscr{S}\left(\mathbb{R}^{N}\right)$, all $r>0$ and all $x \in \mathbb{R}^{N}$

$$
\begin{aligned}
(-\Delta)^{\frac{s}{2}} u(x)= & -C(N, s) \int_{\{|z| \geq r\}} \frac{u(x+z)-u(x)}{|z|^{N+s}} \mathrm{~d} z \\
& -C(N, s) \int_{\{|z| \leq r\}} \frac{u(x+z)-u(x)-\nabla u(x) \cdot z}{|z|^{N+s}} \mathrm{~d} z .
\end{aligned}
$$

Proof. See [12], Theorem 1.

Remark 1. With Proposition 1 it is possible to extend the definition of the fractional Laplacian in (2) for all $u \in C_{b}^{2}\left(\mathbb{R}^{N}\right)$. Furthermore, from a wellknown nonlocal integration-by-parts formula (see, e.g., [8] Lemma A.2), we get

$$
\int_{\mathbb{R}^{N}}(-\Delta)^{\frac{s}{2}} u(x) \varphi(x) \mathrm{d} x=\frac{C(N, s)}{2} \int_{\mathbb{R}^{N}} \int_{\mathbb{R}^{N}} \frac{(u(x)-u(y))(\varphi(x)-\varphi(y))}{|x-y|^{N+s}} \mathrm{~d} x \mathrm{~d} y,
$$

for any $\varphi \in \mathscr{D}\left(\mathbb{R}^{N}\right)$. The right-hand side can also be associated to a bilinear form which is well-defined on $H^{\frac{s}{2}}\left(\mathbb{R}^{N}\right) \times H^{\frac{s}{2}}\left(\mathbb{R}^{N}\right)$. 
A useful convergence result, which we will use later on, can also be found in the work of Droniou and Imbert (see [12], Proposition 1).

Proposition 2. Let $s \in(0,2)$ and $u \in C_{b}^{2}\left(\mathbb{R}^{N}\right)$. If $\left(u_{n}\right)_{n} \subseteq C_{b}^{2}\left(\mathbb{R}^{N}\right)$ is bounded in $L^{\infty}\left(\mathbb{R}^{N}\right)$ and such that $\mathrm{D}^{2} u_{n} \rightarrow \mathrm{D}^{2} u$ locally uniformly in $\mathbb{R}^{N}$ for $n \rightarrow \infty$, then

$$
(-\Delta)^{\frac{s}{2}} u_{n} \rightarrow(-\Delta)^{\frac{s}{2}} u \quad \text { locally uniformly in } \mathbb{R}^{N} \text { for } n \rightarrow \infty \text {. }
$$

Proof. See [12], Proposition 1.

\section{Concept of Solution and Main Results}

Now, we introduce the notion of entropy solutions which is adapted from the work of N. Alibaud (see [1]) and point out where it fits within the well-known concepts of classical and distributional solutions.

Definition 1. A function $u \in L^{\infty}\left(\mathbb{R}^{N}\right)$ is called entropy solution to $\left(\mathscr{P}_{f}\right)$ if for all $r>0$, all $\varphi \in \mathscr{D}\left(\mathbb{R}^{N}\right)$ with $\varphi \geq 0$, all $\eta \in C^{2}(\mathbb{R})$ convex and all $\phi=\left(\phi_{1}, \ldots, \phi_{N}\right)$ with $\phi_{i}^{\prime}=\eta^{\prime} F_{i}^{\prime}$ for $i=1, \ldots, N$ it holds

$$
\begin{aligned}
& \int_{\mathbb{R}^{N}}(f(x)-b(u(x))) \eta^{\prime}(u(x)) \varphi(x)+\phi(u(x)) \cdot \nabla \varphi(x) \mathrm{d} x \\
& \quad+C(N, s) \int_{\mathbb{R}^{N}} \int_{\{|z| \geq r\}} \eta^{\prime}(u(x)) \frac{u(x+z)-u(x)}{|z|^{N+s}} \varphi(x) \mathrm{d} z \mathrm{~d} x \\
& \quad+C(N, s) \int_{\mathbb{R}^{N}} \int_{\{|z| \leq r\}} \eta(u(x)) \frac{\varphi(x+z)-\varphi(x)-\nabla \varphi(x) \cdot z}{|z|^{N+s}} \mathrm{~d} z \mathrm{~d} x \geq 0 .
\end{aligned}
$$

Remark 2. A function $\eta$, as in Definition 1, is called entropy. The corresponding function $\phi$ is called entropy flux and $(\eta, \phi)$ is called entropy-flux-pair.

Proposition 3. (i) Classical solutions to $\left(\mathscr{P}_{f}\right)$, i.e., $u \in C_{b}^{2}\left(\mathbb{R}^{N}\right)$ satisfying the equation $\left(\mathscr{P}_{f}\right)$ pointwise for all $x \in \mathbb{R}^{N}$, are entropy solutions.

(ii) Entropy solutions are distributional solutions in the sense that

$$
\begin{aligned}
& \int_{\mathbb{R}^{N}}(f(x)-b(u(x))) \varphi(x)+F(u(x)) \cdot \nabla \varphi(x)-u(x)(-\Delta)^{\frac{s}{2}} \varphi(x) \mathrm{d} x=0 \\
& \forall \varphi \in \mathscr{D}\left(\mathbb{R}^{N}\right) .
\end{aligned}
$$

Proof. See "Appendix".

The main goal of this paper is to prove the following theorems, concerning the existence and uniqueness of entropy solutions.

Theorem 1. For all $f \in L^{1}\left(\mathbb{R}^{N}\right) \cap L^{\infty}\left(\mathbb{R}^{N}\right)$ there exists an entropy solution $u$ to $\left(\mathscr{P}_{f}\right)$ such that $b(u) \in L^{1}\left(\mathbb{R}^{N}\right)$. 
Theorem 2. For $f, \tilde{f} \in L^{1}\left(\mathbb{R}^{N}\right) \cap L^{\infty}\left(\mathbb{R}^{N}\right)$ let $u, \tilde{u} \in L^{\infty}\left(\mathbb{R}^{N}\right)$ be entropy solutions to $\left(\mathscr{P}_{f}\right)$ and $\left(\mathscr{P}_{\tilde{f}}\right)$ respectively such that $b(u), b(\tilde{u}) \in L^{1}\left(\mathbb{R}^{N}\right)$. Then it holds true that

$$
\int_{\mathbb{R}^{N}}|b(u(x))-b(\tilde{u}(x))| \mathrm{d} x \leq \int_{\mathbb{R}^{N}}|f(x)-\tilde{f}(x)| \mathrm{d} x .
$$

Remark 3. (i) Note that, by the coerciveness condition $\left(\lambda|u|^{2} \leq b(u) \cdot u\right.$ $\forall u \in \mathbb{R})$ on $b$, any entropy solution $u$ of $\left(\mathscr{P}_{f}\right)$ with $b(u) \in L^{1}\left(\mathbb{R}^{\bar{N}}\right)$ already belongs to $L^{1}\left(\mathbb{R}^{N}\right) \cap L^{\infty}\left(\mathbb{R}^{N}\right)$, and then, by the Lipschitz condition on $b$, also $b(u) \in L^{1}\left(\mathbb{R}^{n}\right) \cap L^{\infty}\left(\mathbb{R}^{N}\right)$.

(ii) If $f=\tilde{f}$ almost everywhere, it follows directly from Theorem 2 that $b(u)=b(\tilde{u})$ almost everywhere holds. If the nonlinearity $b$ is strictly monotone, we further obtain $u=\tilde{u}$ almost everywhere. Therefore, we know that the entropy solution $u$ of $\left(\mathscr{P}_{f}\right)$ is unique. In the general case of nondecreasing $b$, the uniqueness of the saturation function $b(u)$ does not imply uniqueness of the entropy solution $u$. It would be interesting to study the uniqueness of the entropy solution $u$ itself, using the Lipschitz continuity of $F$ and the properties of the operator $(-\Delta)^{\frac{s}{2}} u+\operatorname{div}(F(u))$. However, this issue is beyond the scope of our contribution and should be addressed elsewhere.

\section{4. $L^{1}$-Contraction Principle}

In this section, we will prove the $L^{1}$-contraction principle stated in Theorem 2 by applying Kruzhkov's method of doubling variables (see [15]). The $L^{1}$-contraction principle and $L^{1}$-comparison principle (see $(9)$ ) will play an important role in the proof of the existence theorem.

Proof. For all $k \in \mathbb{R}$ we choose the entropy-flux-pair $\left(\eta_{k}, \phi_{k}\right)$, which is defined by

$$
\begin{aligned}
\eta_{k}(a) & =|a-k|, \\
\phi_{k}(a) & =\int_{k}^{a} \operatorname{sign}(\tau-k) F^{\prime}(\tau) \mathrm{d} \tau
\end{aligned}
$$

for all $a \in \mathbb{R}$. Since these entropies are not smooth enough, we have to show first that the entropy inequality (5) also holds for this entropy-flux-pair. To this end, we approximate $\eta_{k}$ in the following way:

$$
\eta_{k}^{n}: \mathbb{R} \rightarrow \mathbb{R}, \quad a \mapsto \int_{k}^{a} \varrho^{n}(\sigma-k) \mathrm{d} \sigma
$$


with

$$
\varrho^{n}: \mathbb{R} \rightarrow \mathbb{R}, \quad a \mapsto \begin{cases}1 & \text { for } a>\frac{1}{n} \\ \sin \left(n a \frac{\pi}{2}\right) & \text { for }-\frac{1}{n} \leq a \leq \frac{1}{n} \\ -1 & \text { for } a<-\frac{1}{n} .\end{cases}
$$

Then, for all $n \in \mathbb{N}, \eta_{k}^{n} \in C^{2}(\mathbb{R})$ is convex and such that $\left(\eta_{k}^{n}\right)^{\prime \prime}$ has compact support. Now we can use them in the entropy inequality (5) and prove, with the help of Lebesgue's dominated convergence theorem, that the entropy inequality (5) holds for the pair $\left(\eta_{k}, \phi_{k}\right)$. We can now apply the method of doubling variables.

For the entropy solution $u$ of $\left(\mathscr{P}_{f}\right)$ let $y \in \mathbb{R}^{N}$ be fixed but arbitrary and choose $\varphi(x)=\varphi_{y}(x)=\psi(x, y)$ with $\psi \in \mathscr{D}\left(\mathbb{R}^{N} \times \mathbb{R}^{N}\right), \psi \geq 0$ and $\eta=\eta_{k}$ with $k=\tilde{u}(y)$. If we apply these in (5) and integrate with respect to $y$ over $\mathbb{R}^{N}$, we get

$$
\begin{aligned}
0 \leq & \int_{\mathbb{R}^{N}} \int_{\mathbb{R}^{N}}(f(x)-b(u(x))) \operatorname{sign}(u(x)-\tilde{u}(y)) \psi(x, y) \\
& +\zeta(u(x), \tilde{u}(y)) \cdot \nabla_{x} \psi(x, y) \mathrm{d} y \mathrm{~d} x \\
& +C(N, s)\left(\int_{\mathbb{R}^{N}} \int_{\mathbb{R}^{N}} \int_{\{|z| \geq r\}} \operatorname{sign}(u(x)-\tilde{u}(y)) \frac{u(x+z)-u(x)}{|z|^{N+s}} \psi(x, y) \mathrm{d} z \mathrm{~d} y \mathrm{~d} x\right. \\
& \left.+\int_{\mathbb{R}^{N}} \int_{\mathbb{R}^{N}} \int_{\{|z| \leq r\}}|u(x)-\tilde{u}(y)| \frac{\psi(x+z, y)-\psi(x, y)-\nabla_{x} \psi(x, y) \cdot z}{|z|^{N+s}} \mathrm{~d} z \mathrm{~d} y \mathrm{~d} x\right),
\end{aligned}
$$

where the symmetric function $\zeta$ is given by $\zeta(a, b)=F(\max \{a, b\})-$ $F(\min \{a, b\})$. Analogously, for the entropy solution $\tilde{u}$ of $\left(\mathscr{P}_{\tilde{f}}\right)$ and $x \in \mathbb{R}^{N}$ fixed but arbitrary, we choose $\varphi(y)=\varphi_{x}(y)=\psi(x, y)$ as above, $\eta=\eta_{k}$ with $k=u(x)$, apply these in (5) and integrate with respect to $x$ over $\mathbb{R}^{N}$. If we add these inequalities, we get

$$
\begin{aligned}
0 \leq & \int_{\mathbb{R}^{N}} \int_{\mathbb{R}^{N}}(f(x)-\tilde{f}(y)) \operatorname{sign}(u(x)-\tilde{u}(y)) \psi(x, y)-|b(u(x))-b(\tilde{u}(y))| \psi(x, y) \\
& +\zeta(u(x), \tilde{u}(y)) \cdot\left(\nabla_{x}+\nabla_{y}\right) \psi(x, y) \mathrm{d} y \mathrm{~d} x \\
& +C(N, s) \iint_{\mathbb{R}^{N}} \int_{\mathbb{R}^{N}} \frac{|u(x+z)-\tilde{u}(y+z)|-|u(x)-\tilde{u}(y)|}{|z|^{N+s}} \psi(x, y) \mathrm{d} z \mathrm{~d} y \mathrm{~d} x \\
& +C(N, s) \int_{\mathbb{R}^{N}} \int_{\mathbb{R}^{N}} \int_{\{|z| \leq r\}}|u(x)-\tilde{u}(y)| \\
& \times \frac{\psi(x+z, y)+\psi(x, y+z)-2 \psi(x, y)-\left(\nabla_{x}+\nabla_{y}\right) \psi(x, y) \cdot z}{|z|^{N+s}} \mathrm{~d} z \mathrm{~d} y \mathrm{~d} x .
\end{aligned}
$$

In the next step we pass to the limit with $r \rightarrow 0$. 
Lemma 1. Under the assumptions of Theorem 2, there holds

$$
\begin{aligned}
& \lim _{r \rightarrow 0} C(N, s) \int_{\mathbb{R}^{N}} \int_{\mathbb{R}^{N}} \int_{\{|z| \leq r\}}|u(x)-\tilde{u}(y)| \\
& \quad \times \frac{\psi(x+z, y)+\psi(x, y+z)-2 \psi(x, y)-\left(\nabla_{x}+\nabla_{y}\right) \psi(x, y) \cdot z}{|z|^{N+s}} \mathrm{~d} z \mathrm{~d} y \mathrm{~d} x=0 .
\end{aligned}
$$

Proof. Without loss of generality let $r<1$. We show that the integrand belongs to $L^{1}\left(\mathbb{R}^{N} \times \mathbb{R}^{N} \times \overline{B_{1}(0)}\right)$. The claim then follows by Lebesgue's dominated convergence theorem. Similar to the proof of Theorem 3(i) we get

$$
\begin{aligned}
& |u(x)-\tilde{u}(y)| \frac{\left|\psi(x+z, y)-\psi(x, y)-\nabla_{x} \psi(x, y) \cdot z\right|}{|z|^{N+s}} \\
& \quad \leq\left(\|u\|_{\infty}+\|\tilde{u}\|_{\infty}\right) \int_{0}^{1}(1-\tau) \frac{\left|D^{2} \psi(x+\tau z, y)\right|}{|z|^{N+s-2}} \mathrm{~d} \tau \\
& \quad \leq C\left(\|u\|_{\infty},\|\tilde{u}\|_{\infty},\left\|\mathrm{D}^{2}(\psi)\right\|_{\infty}\right) \frac{\chi_{\operatorname{supp}(\psi)+\overline{B_{1}(0,0)}}(x, y)}{|z|^{N+s-2}} \in L^{1}\left(\mathbb{R}^{N} \times \mathbb{R}^{N} \times \overline{B_{1}(0)}\right) .
\end{aligned}
$$

An analogous calculation for the term

$$
|u(x)-\tilde{u}(y)| \frac{\left|\psi(x, y+z)-\psi(x, y)-\nabla_{y} \psi(x, y) \cdot z\right|}{|z|^{N+s}}
$$

completes the proof of the Lemma.

With similar calculations we can also pass to the limit with $r \rightarrow 0$ in the regular term of the nonlocality. If we combine these results, we obtain, for $r \rightarrow 0$ :

$$
\begin{aligned}
0 \leq & \int_{\mathbb{R}^{N}} \int_{\mathbb{R}^{N}}(f(x)-\tilde{f}(y)) \operatorname{sign}(u(x)-\tilde{u}(y)) \psi(x, y)-|b(u(x))-b(\tilde{u}(y))| \psi(x, y) \\
& +\zeta(u(x), \tilde{u}(y)) \cdot\left(\nabla_{x}+\nabla_{y}\right) \psi(x, y) \mathrm{d} y \mathrm{~d} x \\
& +\int_{\mathbb{R}^{N}} \int_{\mathbb{R}^{N}}|u(x)-\tilde{u}(y)|\left(C(N, s) \int_{\{|z| \geq 1\}} \frac{\psi(x+z, y+z)-\psi(x, y)}{|z|^{N+s}} \mathrm{~d} z\right. \\
& \left.+C(N, s) \int_{\{|z| \leq 1\}} \frac{\psi(x+z, y+z)-\psi(x, y)-\left(\nabla_{x}+\nabla_{y}\right) \psi(x, y) \cdot z}{|z|^{N+s}} \mathrm{~d} z\right) \mathrm{d} y \mathrm{~d} x .
\end{aligned}
$$

We now choose $\mu>0, \psi(x, y)=\rho_{\mu}(y-x) \Phi(x)$, where $\rho_{\mu} \in \mathscr{D}\left(B_{\mu}(0)\right)$ with $\rho_{\mu} \geq 0$ such that $\int_{\mathbb{R}^{N}} \rho_{\mu}(z) \mathrm{d} z=1$ and $\Phi \in \mathscr{D}\left(\mathbb{R}^{N}\right)$ with $\Phi \geq 0$. Then we have

$$
\begin{aligned}
0 \leq & \int_{\mathbb{R}^{N}} \int_{\mathbb{R}^{N}}|f(x)-\tilde{f}(y)| \rho_{\mu}(y-x) \Phi(x)-|b(u(x))-b(\tilde{u}(y))| \rho_{\mu}(y-x) \Phi(x) \\
& +|u(x)-\tilde{u}(y)| \rho_{\mu}(y-x) \Xi(x) \mathrm{d} y \mathrm{~d} x=: I_{\mu},
\end{aligned}
$$


where $\Xi(x):=L|\nabla \Phi(x)|-(-\Delta)^{\frac{s}{2}} \Phi(x)$ and $L$ is the Lipschitz constant of $F$ on the set $[-m, m]$ with $m=\max \left\{\|u\|_{\infty},\|\tilde{u}\|_{\infty}\right\}$. Letting $\mu \rightarrow 0$, we can show that

$$
0 \leq \int_{\mathbb{R}^{N}}|f(x)-\tilde{f}(x)| \Phi(x)-|b(u(x))-b(\tilde{u}(x))| \Phi(x)+|u(x)-\tilde{u}(x)| \Xi(x) \mathrm{d} x
$$

holds. Now, choose $\Phi \in \mathscr{D}\left(\mathbb{R}^{N}\right), 0 \leq \Phi \leq 1$, such that

$$
\Phi(x)= \begin{cases}1 & \text { if }\|x\| \leq 1 \\ 0 & \text { if }\|x\| \geq 2\end{cases}
$$

Then we define $\Phi_{n}(x)=\Phi\left(\frac{x}{n}\right) \forall n \in \mathbb{N}$. It yields $D^{2} \Phi_{n} \rightarrow 0$ pointwise and locally uniformly in $\mathbb{R}^{N}$ for $n \rightarrow \infty$. For $\Phi=\Phi_{n}$ in (7), we can make use of Proposition 2, the conditions on $b$ (see Remark 3(i)), which guarantee that $|u-\tilde{u}| \in L^{1}\left(\mathbb{R}^{N}\right)$, and Lebesgue's theorem of dominated convergence to get

$$
\begin{aligned}
0 \leq & \lim _{n \rightarrow \infty} \int_{\mathbb{R}^{N}}|f(x)-\tilde{f}(x)| \Phi_{n}(x)-|b(u(x))-b(\tilde{u}(x))| \Phi_{n}(x) \\
& +|u(x)-\tilde{u}(x)|\left(L\left|\nabla \Phi_{n}(x)\right|-(-\Delta)^{\frac{s}{2}} \Phi_{n}(x)\right) \mathrm{d} x \\
= & \int_{\mathbb{R}^{N}}|f(x)-\tilde{f}(x)|-|b(u(x))-b(\tilde{u}(x))| \mathrm{d} x .
\end{aligned}
$$

This completes the proof of Theorem 2 .

\subsection{Extensions and Remarks}

Remark 4. Similar to the proof of Theorem 2, we can show the following $L^{1}$ comparison principles:

$$
\int_{\mathbb{R}^{N}}(b(u(x))-b(\tilde{u}(x)))^{+} \mathrm{d} x \leq \int_{\mathbb{R}^{N}}(f(x)-\tilde{f}(x))^{+} \mathrm{d} x
$$

and

$$
\int_{\mathbb{R}^{N}}(b(u(x))-b(\tilde{u}(x)))^{-} \mathrm{d} x \leq \int_{\mathbb{R}^{N}}(f(x)-\tilde{f}(x))^{-} \mathrm{d} x .
$$

To prove this, we apply the method of doubling variables again, but with entropies

$$
\eta_{k}(a)=(a-k)^{+}
$$

and

$$
\eta_{k}(a)=(a-k)^{-} .
$$

respectively. 
Remark 5. (i) Let $f, \tilde{f}, u, \tilde{u} \in L^{\infty}\left(\mathbb{R}^{N}\right)$ be such that $u$ satisfies the entropy inequality $(5)$ of $\left(\mathscr{P}_{f}\right)$ and $\tilde{u}$ of $\left(\mathscr{P}_{\tilde{f}}\right)$ respectively. Then we can still prove the ,local" inequality (7) for the contraction and comparison principle.

(ii) If $u$ is an entropy solution to $\left(\mathscr{P}_{f}\right)$ with $b(u) \in L^{1}\left(\mathbb{R}^{N}\right)$ and $k>0$ then $(u-k)^{+} \in L^{1}\left(\mathbb{R}^{N}\right)$ and, since $\tilde{u} \equiv k$ is a classical solution to $\left(\mathscr{P}_{\tilde{f}}\right)$ with $\tilde{f} \equiv b(k)$, we can also pass to the limit in (8) for the comparison principle, to show that (9) holds with $\tilde{u}=\tilde{f}=k$.

We are now able to prove the following $L^{\infty}$-estimate.

Lemma 2. Let $f \in L^{1}\left(\mathbb{R}^{N}\right) \cap L^{\infty}\left(\mathbb{R}^{N}\right)$ and $u$ be an entropy solution to $\left(\mathscr{P}_{f}\right)$, then we have

$$
\|b(u)\|_{\infty} \leq\|f\|_{\infty}
$$

Proof. Since $b$ satisfies the growth condition, $b$ is surjective, i.e., for $\left\|f^{+}\right\|_{\infty} \in \mathbb{R}$ there exists $c \in \mathbb{R}, c \geq 0$, such that $b(c)=\left\|f^{+}\right\|_{\infty}$. Let $\tilde{f} \equiv\left\|f^{+}\right\|_{\infty}$ and $\tilde{u} \equiv c$, then it follows that $b(u) \leq\left\|f^{+}\right\|_{\infty}$ almost everywhere with Remark 5 (ii). Analogously we can show that $-\left\|f^{-}\right\|_{\infty} \leq b(u)$ almost everywhere which completes the proof.

\section{Existence of Entropy Solutions}

\subsection{The Vanishing Viscosity Method}

We are now turning our attention to the existence proof for entropy solutions to problem $\left(\mathscr{P}_{f}\right)$. For this, consider the following modified problems for $\varepsilon>0$ :

$$
b(u)+\varepsilon u-\varepsilon \Delta u+(-\Delta)^{\frac{s}{2}} u=g
$$

with $g \in L^{2}\left(\mathbb{R}^{N}\right)$. Assume $u \in C_{b}^{2}\left(\mathbb{R}^{N}\right) \cap H^{1}\left(\mathbb{R}^{N}\right)$ to be a classical solution to $\left(\tilde{\mathscr{P}}_{g}^{\varepsilon}\right)$. We obtain a weak formulation of $\left(\tilde{\mathscr{P}}_{g}^{\varepsilon}\right)$ by multiplication with a test function $\nu \in \mathscr{D}\left(\mathbb{R}^{N}\right)$ and subsequent integration over $\mathbb{R}^{N}$ where we can allow for right-hand sides in $H^{-1}\left(\mathbb{R}^{N}\right)$ :

Definition 2. Let $g \in H^{-1}\left(\mathbb{R}^{N}\right)$. A function $u \in H^{1}\left(\mathbb{R}^{N}\right)$ is called weak solution to $\left(\tilde{\mathscr{P}}_{g}^{\varepsilon}\right)$, if

$$
\begin{aligned}
& \int_{\mathbb{R}^{N}} b(u(x)) \nu(x) \mathrm{d} x+\varepsilon \int_{\mathbb{R}^{N}} u(x) \nu(x) \mathrm{d} x+\varepsilon \int_{\mathbb{R}^{N}} \nabla u(x) \nabla \nu(x) \mathrm{d} x \\
& \left.\quad+\frac{C(N, s)}{2} \int_{\mathbb{R}^{N}} \int_{\mathbb{R}^{N}} \frac{(u(x)-u(y))(\nu(x)-\nu(y))}{|x-y|^{N+s}} \mathrm{~d} x \mathrm{~d} y=<g, \nu\right\rangle_{H^{-1}, H^{1}}
\end{aligned}
$$

for all $\nu \in H^{1}\left(\mathbb{R}^{N}\right)$.

Remark 6. Since $H^{1}\left(\mathbb{R}^{N}\right) \hookrightarrow H^{\frac{s}{2}}\left(\mathbb{R}^{N}\right)$ (see [9] Corollaire 4.34 (ii)), all integrals are well-defined. 
First, we want to prove the existence and uniqueness of weak solutions to the modified problem $\left(\tilde{\mathscr{P}}_{g}^{\varepsilon}\right)$ for an arbitrary right-hand side $g \in H^{-1}\left(\mathbb{R}^{N}\right)$ with the help of Zarantonello's theorem (see [13], Theorem 3.5.2). By a fixedpoint argument, we will then prove that there exist also weak solutions to the modified, doubly nonlinear problem $\left(\mathscr{P}_{f}^{\varepsilon}\right)$ with right-hand side $f \in L^{2}\left(\mathbb{R}^{N}\right)$ which we will define later on. Based on the higher regularity of these solutions and on the monotonicity of the term $\varepsilon u-\varepsilon \Delta u$ we can prove that these weak solutions are already entropy solutions. Finally, we will show that the weak solutions to the modified, doubly nonlinear problems converge to the entropy solution of problem $\left(\mathscr{P}_{f}\right)$.

Proposition 4. For all $g \in H^{-1}\left(\mathbb{R}^{N}\right)$ there exists a unique weak solution $u \in$ $H^{1}\left(\mathbb{R}^{N}\right)$ to $(\tilde{\mathscr{P}} \varepsilon)$.

Proof. We define

$$
\begin{aligned}
& a: H^{1}\left(\mathbb{R}^{N}\right) \times H^{1}\left(\mathbb{R}^{N}\right) \rightarrow \mathbb{R} \\
& (u, v) \mapsto \int_{\mathbb{R}^{N}} b(u(x)) v(x) \mathrm{d} x+\varepsilon \int_{\mathbb{R}^{N}} u(x) v(x) \mathrm{d} x+\varepsilon \int_{\mathbb{R}^{N}} \nabla u(x) \cdot \nabla v(x) \mathrm{d} x \\
& \quad+\frac{C(N, s)}{2} \int_{\mathbb{R}^{N}} \int_{\mathbb{R}^{N}} \frac{(u(x)-u(y))(v(x)-v(y))}{|x-y|^{N+s}} \mathrm{~d} x \mathrm{~d} y .
\end{aligned}
$$

Then $a(u, \cdot)$ is linear and bounded for every fixed $u \in H^{1}\left(\mathbb{R}^{N}\right)$, i.e., it is an element in $H^{-1}\left(\mathbb{R}^{N}\right)$. Consider now

$$
\begin{aligned}
\mathscr{A}: H^{1}\left(\mathbb{R}^{N}\right) & \rightarrow H^{-1}\left(\mathbb{R}^{N}\right) \\
u & \mapsto a(u, \cdot)
\end{aligned}
$$

and we claim that $\mathscr{A}$ is Lipschitz continuous and strongly monotone. It then follows by the Theorem of Zarantonello that $\mathscr{A}$ is bijective and therefore, for all $g \in H^{-1}\left(\mathbb{R}^{N}\right)$, there exists a unique $u \in H^{1}\left(\mathbb{R}^{N}\right)$ such that

$$
a(u, v)=<g, v>_{H^{-1}, H^{1}}
$$

for all $v \in H^{1}\left(\mathbb{R}^{N}\right)$. This completes the proof.

Let $u_{1}, u_{2} \in H^{1}\left(\mathbb{R}^{N}\right)$. Then it holds

$$
\begin{aligned}
& \left\|\mathscr{A} u_{1}-\mathscr{A} u_{2}\right\|_{H^{-1}} \\
& =\sup _{\|v\|_{H^{1}} \leq 1}\left|<\mathscr{A} u_{1}-\mathscr{A} u_{2}, v>_{H^{-1}, H^{1}}\right| \\
& \leq \sup _{\|v\|_{H^{1}} \leq 1} \int_{\mathbb{R}^{N}}\left|b\left(u_{1}(x)\right)-b\left(u_{2}(x)\right)\right||v(x)| \mathrm{d} x \\
& \quad+\varepsilon \int_{\mathbb{R}^{N}}\left|u_{1}(x)-u_{2}(x)\left\|v(x)\left|\mathrm{d} x+\varepsilon \int_{\mathbb{R}^{N}}\right| \nabla u_{1}(x)-\nabla u_{2}(x)\right\| \nabla v(x)\right| \mathrm{d} x
\end{aligned}
$$




$$
\begin{aligned}
& +\frac{C(N, s)}{2} \int_{\mathbb{R}^{N}} \int_{\mathbb{R}^{N}} \frac{\left|\left(u_{1}(x)-u_{2}(x)\right)-\left(u_{1}(y)-u_{2}(y)\right) \| v(x)-v(y)\right|}{|x-y|^{N+s}} \mathrm{~d} x \mathrm{~d} y \\
\leq & \sup _{\|v\|_{H^{1}} \leq 1} L_{b}\left\|u_{1}-u_{2}\right\|_{L^{2}}\|v\|_{L^{2}}+\varepsilon\left\|u_{1}-u_{2}\right\|_{L^{2}}\|v\|_{L^{2}}+\varepsilon\left\|\nabla u_{1}-\nabla u_{2}\right\|_{L^{2}}\|\nabla v\|_{L^{2}} \\
& +\frac{C(N, s)}{2}\left[u_{1}-u_{2}\right]_{s / 2}[v]_{s / 2} \leq C(N, s, \varepsilon)\left\|u_{1}-u_{2}\right\|_{H^{1}},
\end{aligned}
$$

where $L_{b}$ is the Lipschitz constant of $b$ and therefore, $\mathscr{A}$ is Lipschitz continuous. Moreover,

$$
\begin{aligned}
< & \mathscr{A} u_{1}-\mathscr{A} u_{2}, u_{1}-u_{2}>_{H^{-1}, H^{1}} \\
= & \int_{\mathbb{R}^{N}}\left(b\left(u_{1}(x)\right)-b\left(u_{2}(x)\right)\right)\left(u_{1}(x)-u_{2}(x)\right) \mathrm{d} x \\
& \quad+\varepsilon\left\|u_{1}-u_{2}\right\|_{L^{2}}^{2}+\varepsilon\left\|\nabla u_{1}-\nabla u_{2}\right\|_{L^{2}}^{2}+\frac{C(N, s)}{2}\left[u_{1}-u_{2}\right]_{s / 2}^{2} \\
\geq & \varepsilon\left\|u_{1}-u_{2}\right\|_{H^{1}}^{2},
\end{aligned}
$$

since $b$ is nondecreasing, i.e. $\mathscr{A}$ is also strongly monotone.

If the right-hand side $g$ in problem $\left(\tilde{\mathscr{P}}_{g}^{\varepsilon}\right)$ has better regularity, then also the weak solution $u \in H^{1}\left(\mathbb{R}^{N}\right)$ of $\left(\tilde{\mathscr{P}}_{g}^{\varepsilon}\right)$ has higher regularity. Indeed, one has

Lemma 3. Let $g \in L^{2}\left(\mathbb{R}^{N}\right)$. For the unique weak solution $u \in H^{1}\left(\mathbb{R}^{N}\right)$ to $\left(\tilde{\mathscr{P}}_{g}^{\varepsilon}\right)$ it holds

$$
u \in H_{l o c}^{2}\left(\mathbb{R}^{N}\right) .
$$

Proof. In general, for $0<s \leq 1$, it is known that (see [10], Proposition 3.6)

$$
u \in H^{1}\left(\mathbb{R}^{N}\right) \Rightarrow(-\Delta)^{\frac{s}{2}} u \in L^{2}\left(\mathbb{R}^{N}\right) .
$$

As a consequence, for the unique weak solution to $\left(\tilde{\mathscr{P}}_{g}^{\varepsilon}\right) u \in H^{1}\left(\mathbb{R}^{N}\right)$, it follows that

$$
\varepsilon u-\varepsilon \Delta u=g-(-\Delta)^{\frac{s}{2}} u-b(u) \in L^{2}\left(\mathbb{R}^{N}\right)
$$

in the distributional sense, hence $\Delta u \in L^{2}\left(\mathbb{R}^{N}\right)$ and from classical regularity results for the Laplacian (see e.g. [14], Chapter 6.3.1) it follows that

$$
u \in H_{\mathrm{loc}}^{2}\left(\mathbb{R}^{N}\right) \text {. }
$$

Corollary 1. From Lemma 3 it follows that the weak solution $u$ to $\left(\begin{array}{c}\tilde{P}_{g}^{\varepsilon} \\ \text { ) }\end{array}\right)$ with right-hand side $g \in L^{2}\left(\mathbb{R}^{N}\right)$ satisfies the equation pointwise almost everywhere.

Now, we also want to consider the nonlinearity $\operatorname{div} F(u)$ in our equation. Since we can not show the existence of weak solutions with such a term directly, 
we need to look at approximating problems. To this end, for every $R>0$, let $\varrho_{R} \in C^{\infty}\left(\mathbb{R}^{N}\right)$ be equipped with the following properties:

$$
\begin{aligned}
0 & \leq \varrho_{R} \leq 1, \\
\varrho_{R} & =1 \text { on } B_{R}(0), \\
\operatorname{supp} \varrho_{R} & \Subset B_{2 R}(0), \\
\left\|\nabla \varrho_{R}\right\|_{\infty} & \leq C
\end{aligned}
$$

for a constant $C>0$, independent of $R$. For $f \in L^{2}\left(\mathbb{R}^{N}\right), F_{\varepsilon}=F \circ T_{1 / \varepsilon}$ with $T_{1 / \varepsilon}$ the truncation function at level $1 / \varepsilon$ and $w \in H^{1}\left(\mathbb{R}^{N}\right)$ fixed, but arbitrary, the map

$$
H^{1}\left(\mathbb{R}^{N}\right) \ni v \mapsto \int_{\mathbb{R}^{N}} f(x) v(x) \mathrm{d} x+\int_{\mathbb{R}^{N}} F_{\varepsilon}\left(\varrho_{R}(x) w(x)\right) \nabla\left(\varrho_{R}(x) v(x)\right) \mathrm{d} x
$$

is in $H^{-1}\left(\mathbb{R}^{N}\right)$. If we consider the family of integral equations

$$
\begin{gathered}
\int_{\mathbb{R}^{N}} b(u(x)) v(x) \mathrm{d} x+\varepsilon \int_{\mathbb{R}^{N}} u(x) v(x) \mathrm{d} x+\varepsilon \int_{\mathbb{R}^{N}} \nabla u(x) \nabla v(x) \mathrm{d} x \\
+\frac{C(N, s)}{2} \int_{\mathbb{R}^{N}} \int_{\mathbb{R}^{N}} \frac{(u(x)-u(y))(v(x)-v(y))}{|x-y|^{N+s}} \mathrm{~d} x \mathrm{~d} y \\
=\int_{\mathbb{R}^{N}} f(x) v(x) \mathrm{d} x+\int_{\mathbb{R}^{N}} F_{\varepsilon}\left(\varrho_{R}(x) w(x)\right) \nabla\left(\varrho_{R}(x) v(x)\right) \mathrm{d} x
\end{gathered}
$$

for all $v \in H^{1}\left(\mathbb{R}^{N}\right)$, we already know from Proposition 4 that there exists a unique $u=u_{R, w} \in H^{1}\left(\mathbb{R}^{N}\right)$ which satisfies (12) for all $v \in H^{1}\left(\mathbb{R}^{N}\right)$ and thus, we can define the following map:

$$
\begin{aligned}
\Psi_{R}: H^{1}\left(\mathbb{R}^{N}\right) & \rightarrow H^{1}\left(\mathbb{R}^{N}\right) \\
w & \mapsto \text { the unique solution } u \in H^{1}\left(\mathbb{R}^{N}\right) \text { to }(12) .
\end{aligned}
$$

If we take $u$ itself as a test function in (12) and exploit the properties of $\varrho_{R}$, we get the following a-priori estimate:

$$
\begin{aligned}
\varepsilon\|u\|_{H^{1}}^{2} & \leq\|f\|_{L^{2}}\|u\|_{L^{2}}+C_{\varepsilon, R}\|u\|_{H^{1}} \\
\Rightarrow\|u\|_{H^{1}} & \leq \frac{\|f\|_{L^{2}}+C_{\varepsilon, R}}{\varepsilon}=: K_{\varepsilon, R} .
\end{aligned}
$$

Now, consider the set $M=\left\{u \in H^{1}\left(\mathbb{R}^{N}\right):\|u\|_{H^{1}} \leq K_{\varepsilon, R}\right\}$ which is nonempty, bounded, closed and convex. If we restrict $\Psi_{R}$ to the set $M$ and prove that there exists a fixed-point, we obtain the existence of a solution $u$ to (12) with $w=u$. To achieve this, we have to prove that $\Psi_{R}$ is weakly sequentially continuous, for the claim then follows by the fixed-point theorem of Schauder-Tikhonov (see [17], Corollary 9.7).

Lemma 4. The map $\Psi_{R}: M \rightarrow M$ is weakly sequentially continuous. 
Proof. For all $n \in \mathbb{N}$, let $w_{n}, w \in H^{1}\left(\mathbb{R}^{N}\right)$ such that $w_{n} \rightarrow w$ in $H^{1}\left(\mathbb{R}^{N}\right)$ for $n \rightarrow \infty$. Since the sequence of solutions $u_{n}=\Psi_{R}\left(w_{n}\right)$ is bounded in $H^{1}\left(\mathbb{R}^{N}\right)$, it is sufficient to prove that every weakly convergent subsequence of $\left(u_{n}\right)_{n}$ converges weakly to $\Psi_{R}(w)$. Now, let $\left(u_{n}\right)_{n}$ be a not relabeled subsequence such that $u_{n} \rightarrow u$ in $H^{1}\left(\mathbb{R}^{N}\right)$ for $n \rightarrow \infty$. By continuous and compact embeddings we can assume, without loss of generality, that $\left(w_{n}\right)_{n}$ and $\left(u_{n}\right)_{n}$ converge for $n \rightarrow \infty$ in the following way:

$$
\begin{array}{ll}
w_{n} \longrightarrow w & \text { in } L_{\mathrm{loc}}^{2}\left(\mathbb{R}^{N}\right) \text { and almost everywhere in } \mathbb{R}^{N}, \\
u_{n} \rightarrow u & \text { in } H^{1}\left(\mathbb{R}^{N}\right) \hookrightarrow H^{\frac{s}{2}}\left(\mathbb{R}^{N}\right), \\
u_{n} \longrightarrow u & \text { in } L_{\text {loc }}^{2}\left(\mathbb{R}^{N}\right) \text { and almost everywhere in } \mathbb{R}^{N} .
\end{array}
$$

Since $u_{n}$ is a solution to (12) with $w=w_{n}$, for all $n \in \mathbb{N}$ and all $\varphi \in H^{1}\left(\mathbb{R}^{N}\right)$, we have

$$
\begin{gathered}
\int_{\mathbb{R}^{N}} b\left(u_{n}\right)(x) \varphi(x) \mathrm{d} x+\varepsilon \int_{\mathbb{R}^{N}} u_{n}(x) \varphi(x) \mathrm{d} x+\varepsilon \int_{\mathbb{R}^{N}} \nabla u_{n}(x) \nabla \varphi(x) \mathrm{d} x \\
\quad+\frac{C(N, s)}{2} \int_{\mathbb{R}^{N}} \int_{\mathbb{R}^{N}} \frac{\left(u_{n}(x)-u_{n}(y)\right)(\varphi(x)-\varphi(y))}{|x-y|^{N+s}} \mathrm{~d} x \mathrm{~d} y \\
=\int_{\mathbb{R}^{N}} f(x) \varphi(x) \mathrm{d} x+\int_{\mathbb{R}^{N}} F_{\varepsilon}\left(\varrho_{R}(x) w_{n}\right)(x) \nabla\left(\varrho_{R}(x) \varphi(x)\right) \mathrm{d} x .
\end{gathered}
$$

Thanks to (13)-(15) and as $b$ as well as $F_{\varepsilon}$ are Lipschitz continuous, we can pass to the limit in all integrals and obtain $u=\Psi_{R}(w)$.

We have therefore proved that, for all $R>0$, there exists $u=u_{R} \in$ $H^{1}\left(\mathbb{R}^{N}\right)$ such that

$$
\begin{aligned}
\int_{\mathbb{R}^{N}} b(u(x)) v(x) \mathrm{d} x+\varepsilon \int_{\mathbb{R}^{N}} u(x) v(x) \mathrm{d} x+\varepsilon \int_{\mathbb{R}^{N}} \nabla u(x) \nabla v(x) \mathrm{d} x \\
\quad+\frac{C(N, s)}{2} \int_{\mathbb{R}^{N}} \int_{\mathbb{R}^{N}} \frac{(u(x)-u(y))(v(x)-v(y))}{|x-y|^{N+s}} \mathrm{~d} x \mathrm{~d} y \\
=\int_{\mathbb{R}^{N}} f(x) v(x) \mathrm{d} x+\int_{\mathbb{R}^{N}} F_{\varepsilon}\left(\varrho_{R}(x) u(x)\right) \nabla\left(\varrho_{R}(x) v(x)\right) \mathrm{d} x
\end{aligned}
$$

for all $v \in H^{1}\left(\mathbb{R}^{N}\right)$. Our next goal is to pass to the limit with $R \rightarrow \infty$. First, we need a technical result which allows us to get rid of the convection term. From the divergence theorem of Gauss we can show the following Lemma.

Lemma 5. For every $u \in H^{1}\left(\mathbb{R}^{N}\right)$ it holds

$$
\int_{\mathbb{R}^{N}} F_{\varepsilon}(u(x)) \nabla u(x) \mathrm{d} x=0 .
$$


Proof. See "Appendix".

Hence, if we apply $\nu=u_{R} \in H^{1}\left(\mathbb{R}^{N}\right)$ as a test function in (16) we get

$$
\left\|u_{R}\right\|_{H^{1}} \leq \frac{\|f\|_{L^{2}}}{\varepsilon}
$$

for all $R>0$, where we used Lemma 5 . Thus, there exists a subsequence, still denoted by $\left(u_{R}\right)_{R}$, such that

$$
\begin{aligned}
& u_{R} \rightarrow u \text { in } H^{1}\left(\mathbb{R}^{N}\right), \\
& u_{R} \rightarrow u \text { in } L_{l o c}^{2}\left(\mathbb{R}^{N}\right) \text { and almost everywhere in } \mathbb{R}^{N}
\end{aligned}
$$

for $R \rightarrow \infty$. For $v \in C_{c}^{\infty}\left(\mathbb{R}^{N}\right)$ we can now pass to the limit with $R \rightarrow \infty$ in (16): If $R$ is large enough such that $\operatorname{supp} v \subseteq B_{R}(0)$, it yields

$$
\int_{\mathbb{R}^{N}} F_{\varepsilon}\left(\varrho_{R}(x) u_{R}(x)\right) \nabla\left(\varrho_{R}(x) v(x)\right) \mathrm{d} x=\int_{\operatorname{supp} v} F_{\varepsilon}\left(u_{R}(x)\right) \nabla v(x) \mathrm{d} x
$$

and on $\operatorname{supp} v \subseteq B_{R}(0)$ it holds

$$
F_{\varepsilon}\left(\varrho_{R}(x) u_{R}(x)\right) \nabla\left(\varrho_{R}(x) v(x)\right) \rightarrow F_{\varepsilon}(u(x)) \nabla v(x)
$$

almost everywhere for $R \rightarrow \infty$ and

$$
\left|F_{\varepsilon}\left(\varrho_{R}(x) u_{R}(x)\right) \nabla\left(\varrho_{R}(x) v(x)\right)\right| \leq\left\|F_{\varepsilon}\right\|_{\infty}|\nabla v(x)| \in L^{1}(\operatorname{supp} v) .
$$

Therefore, we can pass to the limit with $R \rightarrow \infty$ in (16) (with $u=u_{R}$ ) and obtain a distributional solution in the sense of Definition 2 with $g=f-$ $\operatorname{div} F(u)$ and test functions $\nu \in C_{c}^{\infty}\left(\mathbb{R}^{N}\right)$. By density of $C_{c}^{\infty}\left(\mathbb{R}^{N}\right)$ in $H^{1}\left(\mathbb{R}^{N}\right)$ it follows that $u$ is the desired weak solution of

$$
b(u)+\varepsilon u-\varepsilon \Delta u+\operatorname{div} F_{\varepsilon}(u)+(-\Delta)^{\frac{s}{2}} u=f
$$

for all $\varepsilon>0$ and all $f \in L^{1}\left(\mathbb{R}^{N}\right) \cap L^{\infty}\left(\mathbb{R}^{N}\right)$.

Proposition 5. For the weak solution $u \in H^{1}\left(\mathbb{R}^{N}\right)$ to $\left(\mathscr{P}_{f}^{\varepsilon}\right)$ with $f \in L^{1}\left(\mathbb{R}^{N}\right) \cap$ $L^{\infty}\left(\mathbb{R}^{N}\right)$ the following holds:

(i) $\|b(u)\|_{L^{1}} \leq\|f\|_{L^{1}},\|u\|_{L^{1}} \leq \frac{1}{\lambda}\|f\|_{L^{1}}$.

(ii) $\|u\|_{L^{\infty}} \leq \frac{1}{\lambda}\|f\|_{L^{\infty}}$.

Proof. (i) Let $k>0$. Applying the test function $\frac{1}{k} T_{k}(u) \in H^{1}\left(\mathbb{R}^{N}\right)$ in the weak formulation of $\left(\mathscr{P}_{f}^{\varepsilon}\right)$, we get

$$
\begin{aligned}
& \int_{\mathbb{R}^{N}} f(x) \frac{1}{k} T_{k}(u(x)) \mathrm{d} x=\int_{\mathbb{R}^{N}} b(u(x)) \frac{1}{k} T_{k}(u(x)) \mathrm{d} x+\frac{\varepsilon}{k} \int_{\mathbb{R}^{N}} u(x) T_{k}(u(x)) \mathrm{d} x \\
& +\frac{\varepsilon}{k} \int_{\{|u| \leq k\}}|\nabla u(x)|^{2} \mathrm{~d} x-\int_{\{|u| \leq k\}} F_{\varepsilon}(u(x)) \cdot \nabla u(x) \mathrm{d} x \\
& \quad+\frac{C(N, s)}{2 k} \int_{\mathbb{R}^{N}} \int_{\mathbb{R}^{N}} \frac{(u(x)-u(y))\left(T_{k}(u(x))-T_{k}(u(y))\right)}{|x-y|^{N+s}} \mathrm{~d} x \mathrm{~d} y .
\end{aligned}
$$


If we use Lemma 5 and the positivity of the terms on the left-hand side, we obtain

$$
\int_{\mathbb{R}^{N}} b(u(x)) \frac{1}{k} T_{k}(u(x)) \mathrm{d} x \leq\|f\|_{L^{1}} .
$$

Since for the test function it holds $\frac{1}{k} T_{k}(u) \rightarrow \operatorname{sign} u$ almost everywhere in $\mathbb{R}^{N}$ for $k \rightarrow 0$, with Fatou's Lemma we get that

$$
\begin{aligned}
\|b(u)\|_{L^{1}} & =\int_{\mathbb{R}^{N}}|b(u(x))| \mathrm{d} x=\int_{\mathbb{R}^{N}} b(u(x)) \operatorname{sign} b(u(x)) \mathrm{d} x \\
& \leq \liminf _{k \rightarrow 0} \int_{\mathbb{R}^{N}} b(u(x)) \frac{1}{k} T_{k}(u(x)) \mathrm{d} x \leq\|f\|_{L^{1}} .
\end{aligned}
$$

This implies $b(u) \in L^{1}\left(\mathbb{R}^{N}\right)$ and thanks to the coerciveness condition $(|b(u)| \geq \lambda|u| \forall u \in \mathbb{R})$ of $b$ also $u \in L^{1}\left(\mathbb{R}^{N}\right)$, hence $\|u\|_{L^{1}} \leq \frac{1}{\lambda}\|f\|_{L^{1}}$.

(ii) Let now $k, l>0$. This time, we use the test function $\frac{1}{k} T_{k}^{+}(u-l) \in H^{1}\left(\mathbb{R}^{N}\right)$ in the weak formulation of $\left(\mathscr{P}_{f}^{\varepsilon}\right)$. We then get

$$
\begin{aligned}
\int_{\mathbb{R}^{N}} b & (u(x)) \frac{1}{k} T_{k}^{+}(u(x)-l) \mathrm{d} x+\frac{\varepsilon}{k} \int_{\mathbb{R}^{N}} u(x) T_{k}^{+}(u(x)-l) \mathrm{d} x \\
& +\frac{\varepsilon}{k} \int_{\{l<u<l+k\}}|\nabla u(x)|^{2} \mathrm{~d} x-\int_{\{l<u<l+k\}} F_{\varepsilon}(u(x)) \cdot \nabla u(x) \mathrm{d} x \\
& +\frac{C(N, s)}{2 k} \int_{\mathbb{R}^{N}} \int_{\mathbb{R}^{N}} \frac{(u(x)-u(y))\left(T_{k}^{+}(u(x)-l)-T_{k}^{+}(u(y)-l)\right)}{|x-y|^{N+s}} \mathrm{~d} x \mathrm{~d} y \\
= & \int_{\mathbb{R}^{N}} f(x) \frac{1}{k} T_{k}^{+}(u(x)-l) \mathrm{d} x .
\end{aligned}
$$

With the positivity of the integrands, Lemma 5 and the growth condition of $b$, we obtain

$$
\begin{aligned}
& \lambda \int_{\mathbb{R}^{N}} u(x) \frac{1}{k} T_{k}^{+}(u(x)-l) \mathrm{d} x \leq \int_{\mathbb{R}^{N}} f(x) \frac{1}{k} T_{k}^{+}(u(x)-l) \mathrm{d} x \\
& \quad \Rightarrow \lambda \int_{\mathbb{R}^{N}}(u(x)-l) \frac{1}{k} T_{k}^{+}(u(x)-l) \mathrm{d} x \leq \int_{\mathbb{R}^{N}}(f(x)-\lambda l) \frac{1}{k} T_{k}^{+}(u(x)-l) \mathrm{d} x .
\end{aligned}
$$

Let $l \geq \frac{\left\|f^{+}\right\|_{\infty}}{\lambda}$, we then get for $k \rightarrow 0$ :

$$
\lambda \int_{\mathbb{R}^{N}}(u(x)-l)^{+} \mathrm{d} x \leq \int_{\mathbb{R}^{N}}(f(x)-\lambda l) \operatorname{sign}^{+}(u(x)-l) \mathrm{d} x \leq 0 .
$$


Since the left integrand is positive, it follows that $(u-l)^{+}=0$ almost everywhere in $\mathbb{R}^{N}$ and therefore $u \leq l$ almost everywhere in $\mathbb{R}^{N}$. Analogously, we can show that for $\tilde{l} \leq \frac{-\left\|f^{-}\right\|_{\infty}}{\lambda}$ we have $\tilde{l} \leq u$ almost everywhere in $\mathbb{R}^{N}$. Thus, the claim follows.

Remark \%. For any $\varepsilon>0$, the unique weak solution $u_{\varepsilon}$ of $\left(\mathscr{P}_{f}^{\varepsilon}\right)$ is in $H^{1}\left(\mathbb{R}^{N}\right)$, thus $b\left(u_{\varepsilon}\right)$ is in $L^{2}\left(\mathbb{R}^{N}\right)$. Moreover, since $F_{\varepsilon}=\left(F_{\varepsilon}^{1}, \ldots, F_{\varepsilon}^{N}\right)$ is Lipschitz continuous, from the chain rule for Sobolev functions it follows that

$$
-\operatorname{div} F_{\varepsilon}\left(u_{\varepsilon}\right)=\sum_{i=1}^{N}\left(F_{\varepsilon}^{i}\right)^{\prime}\left(u_{\varepsilon}\right) \frac{\partial u_{\varepsilon}}{\partial x_{i}} \in L^{2}\left(\mathbb{R}^{N}\right) .
$$

With the same arguments as in the proof of Lemma 3 applied with $g=f-$ $\operatorname{div} F_{\varepsilon}\left(u_{\varepsilon}\right)$, using that $(-\Delta)^{\frac{s}{2}} u_{\varepsilon} \in L^{2}\left(\mathbb{R}^{N}\right)$, we have,

$$
-\Delta u_{\varepsilon}=-u_{\varepsilon}-\frac{1}{\varepsilon}\left[b\left(u_{\varepsilon}\right)+\operatorname{div}\left(F_{\varepsilon}\left(u_{\varepsilon}\right)\right)+(-\Delta)^{\frac{s}{2}} u_{\varepsilon}-f\right]
$$

in $L^{2}\left(\mathbb{R}^{N}\right)$ and we may conclude that $u_{\varepsilon} \in H_{\text {loc }}^{2}\left(\mathbb{R}^{N}\right)$, and, according to Corollary 1 , the equation $\left(\mathscr{P}_{f}^{\varepsilon}\right)$ holds pointwise almost everywhere.

Lemma 6. For any $\varepsilon>0$, the unique weak solution $u_{\varepsilon}$ of $\left(\mathscr{P}_{f}^{\varepsilon}\right)$ satisfies the entropy inequality for all convex entropies $\eta \in C^{2}(\mathbb{R}), \phi_{\varepsilon}=\left(\phi_{\varepsilon}^{1}, \ldots, \phi_{\varepsilon}^{N}\right)$ with $\left(\phi_{\varepsilon}^{i}\right)^{\prime}=\eta^{\prime}\left(F_{\varepsilon}^{i}\right)^{\prime}$ for $i=1, \ldots, N$, all $\varphi \in \mathscr{D}\left(\mathbb{R}^{N}\right)$ with $\varphi \geq 0$, and all $r>0$ :

$$
\begin{aligned}
& \int_{\mathbb{R}^{N}}\left(f(x)-\varepsilon u_{\varepsilon}(x)-b\left(u_{\varepsilon}(x)\right)\right) \eta^{\prime}\left(u_{\varepsilon}(x)\right) \varphi(x) \mathrm{d} x-\varepsilon \int_{\mathbb{R}^{N}} \nabla\left(\eta\left(u_{\varepsilon}(x)\right) \cdot \nabla \varphi(x) \mathrm{d} x\right. \\
& \quad+C(N, s) \int_{\mathbb{R}^{N}} \int_{\{|z| \geq r\}} \eta^{\prime}\left(u_{\varepsilon}(x)\right) \frac{u_{\varepsilon}(x+z)-u_{\varepsilon}(x)}{|z|^{N+s}} \varphi(x) \mathrm{d} z \mathrm{~d} x \\
& \quad+C(N, s) \int_{\mathbb{R}^{N}} \int_{\{|z| \leq r\}} \eta\left(u_{\varepsilon}(x)\right) \frac{\varphi(x+z)-\varphi(x)-\nabla \varphi(x) \cdot z}{|z|^{N+s}} \mathrm{~d} z \mathrm{~d} x \\
& \quad+\int_{\mathbb{R}^{N}} \phi_{\varepsilon}\left(u_{\varepsilon}(x)\right) \cdot \nabla \varphi(x) \mathrm{d} x \geq 0 .
\end{aligned}
$$

Proof. Since the weak solution $u_{\varepsilon}$ of $\left(\mathscr{P}_{f}^{\varepsilon}\right)$ is in $H_{l o c}^{2}\left(\mathbb{R}^{N}\right)$ and by Remark 7 , the equation $\left(\mathscr{P}_{f}^{\varepsilon}\right)$ is satisfied pointwise almost everywhere, similarly to the proof of Proposition 3(i) (see "Appendix"), we are able to show the following inequality for all convex entropies $\eta \in C^{2}(\mathbb{R}), \phi_{\varepsilon}=\left(\phi_{\varepsilon}^{1}, \ldots, \phi_{\varepsilon}^{N}\right)$ with $\left(\phi_{\varepsilon}^{i}\right)^{\prime}=$ $\eta^{\prime}\left(F_{\varepsilon}^{i}\right)^{\prime}$ for $i=1, \ldots, N$, all $\varphi \in \mathscr{D}\left(\mathbb{R}^{N}\right)$ with $\varphi \geq 0$, and all $r>0$ :

$$
\int_{\mathbb{R}^{N}}\left(f(x)-b\left(u_{\varepsilon}(x)\right)\right) \eta^{\prime}\left(u_{\varepsilon}(x)\right) \varphi(x)+\phi\left(u_{\varepsilon}(x)\right) \cdot \nabla \varphi(x) \mathrm{d} x
$$




$$
\begin{aligned}
& +C(N, s) \int_{\mathbb{R}^{N}} \int_{\{|z| \geq r\}} \eta^{\prime}\left(u_{\varepsilon}(x)\right) \frac{u_{\varepsilon}(x+z)-u_{\varepsilon}(x)}{|z|^{N+s}} \varphi(x) \mathrm{d} z \mathrm{~d} x \\
& +C(N, s) \int_{\mathbb{R}^{N}} \int_{\{|z| \leq r\}} \frac{\eta\left(u_{\varepsilon}(x+z)\right)-\eta\left(u_{\varepsilon}(x)\right)-\nabla \eta\left(u_{\varepsilon}(x)\right) \cdot z}{|z|^{N+s}} \varphi(x) \mathrm{d} z \mathrm{~d} x \geq 0 .
\end{aligned}
$$

It is left to show that we can transfer the fractional derivative in the last term on the left-hand side onto the test function $\varphi$. To do this, we can choose a sequence $\left(u_{\varepsilon}^{n}\right)_{n} \subseteq C_{b}^{2}\left(\mathbb{R}^{N}\right)$ such that

$$
\begin{aligned}
u_{\varepsilon}^{n} \rightarrow u_{\varepsilon} & \text { in } L_{l o c}^{2}\left(\mathbb{R}^{N}\right) \text { and a. e. in } \mathbb{R}^{N}, \\
\nabla u_{\varepsilon}^{n} \rightarrow \nabla u_{\varepsilon} & \text { in } L_{l o c}^{2}\left(\mathbb{R}^{N}\right) \text { and a. e. in } \mathbb{R}^{N}, \\
D^{2} u_{\varepsilon}^{n} \rightarrow D^{2} u_{\varepsilon} & \text { in } L_{l o c}^{2}\left(\mathbb{R}^{N}\right) \text { and a. e. in } \mathbb{R}^{N} .
\end{aligned}
$$

From Lebesgue's dominated convergence theorem, the proof of Proposition 3(i) (see "Appendix") on the level of the approximating sequence for every $n \in \mathbb{N}$, and again, Lebesgue's dominated convergence theorem, we have

$$
\begin{aligned}
& \int_{\mathbb{R}^{N}} \int_{\{|z| \leq r\}} \frac{\eta\left(u_{\varepsilon}(x+z)\right)-\eta\left(u_{\varepsilon}(x)\right)-\nabla \eta\left(u_{\varepsilon}(x)\right) \cdot z}{|z|^{N+s}} \varphi(x) \mathrm{d} z \mathrm{~d} x \\
& =\lim _{n \rightarrow \infty} \int_{\mathbb{R}^{N}} \int_{\{|z| \leq r\}} \frac{\eta\left(u_{\varepsilon}^{n}(x+z)\right)-\eta\left(u_{\varepsilon}^{n}(x)\right)-\nabla \eta\left(u_{\varepsilon}^{n}(x)\right) \cdot z}{|z|^{N+s}} \varphi(x) \mathrm{d} z \mathrm{~d} x \\
& =\lim _{n \rightarrow \infty} \int_{\mathbb{R}^{N}} \int_{\{|z| \leq r\}} \eta\left(u_{\varepsilon}^{n}(x)\right) \frac{\varphi(x+z)-\varphi(x)-\nabla \varphi(x) \cdot z}{|z|^{N+s}} \mathrm{~d} z \mathrm{~d} x \\
& =\int_{\mathbb{R}^{N}} \int_{\{|z| \leq r\}} \eta\left(u_{\varepsilon}(x)\right) \frac{\varphi(x+z)-\varphi(x)-\nabla \varphi(x) \cdot z}{|z|^{N+s}} \mathrm{~d} z \mathrm{~d} x .
\end{aligned}
$$

\subsection{Convergence Results for the Approximating Solutions}

In the next step, we want to pass to the limit with $\varepsilon \rightarrow 0$. We apply the weak solution $u=u_{\varepsilon} \in H^{1}\left(\mathbb{R}^{N}\right)$ as a test function and use Lemma 5 to get

$$
\varepsilon\left\|u_{\varepsilon}\right\|_{L^{2}}^{2}+\int_{\mathbb{R}^{N}} b\left(u_{\varepsilon}(x)\right) u_{\varepsilon}(x) \mathrm{d} x+\varepsilon\left\|\nabla u_{\varepsilon}\right\|_{L^{2}}^{2}+\frac{C(N, s)}{2}\left[u_{\varepsilon}\right]_{\frac{s}{2}}^{2} \leq\|f\|_{L^{2}}\left\|u_{\varepsilon}\right\|_{L^{2}} .
$$

Therefore, we get

$$
\lambda \int_{\mathbb{R}^{N}}\left|u_{\varepsilon}(x)\right|^{2} \mathrm{~d} x \leq\|f\|_{L^{2}}\left\|u_{\varepsilon}\right\|_{L^{2}}
$$


and also

$$
\varepsilon\left\|u_{\varepsilon}\right\|_{L^{2}}^{2}+\varepsilon\left\|\nabla u_{\varepsilon}\right\|_{L^{2}}^{2}+\frac{C(N, s)}{2}\left[u_{\varepsilon}\right]_{\frac{s}{2}}^{2} \leq\|f\|_{L^{2}}\left\|u_{\varepsilon}\right\|_{L^{2}} .
$$

This implies

$$
\begin{gathered}
\left(u_{\varepsilon}\right)_{\varepsilon} \text { is bounded in } L^{2}\left(\mathbb{R}^{N}\right), \\
\left(\sqrt{\varepsilon} u_{\varepsilon}\right)_{\varepsilon} \text { is bounded in } L^{2}\left(\mathbb{R}^{N}\right), \\
\left(\sqrt{\varepsilon} \nabla u_{\varepsilon}\right)_{\varepsilon} \text { is bounded in }\left[L^{2}\left(\mathbb{R}^{N}\right)\right]^{N}, \\
\left(u_{\varepsilon}\right)_{\varepsilon} \text { is bounded in } H^{\frac{s}{2}}\left(\mathbb{R}^{N}\right) .
\end{gathered}
$$

From (19)-(22) we get, for a proper subsequence that is still denoted by $\left(u_{\varepsilon}\right)_{\varepsilon}$, the following convergence results for $\varepsilon \rightarrow 0$ :

$$
\begin{aligned}
u_{\varepsilon} \rightarrow u & \text { in } H^{\frac{s}{2}}\left(\mathbb{R}^{N}\right) \\
\varepsilon u_{\varepsilon} \rightarrow 0 & \text { in } L^{2}\left(\mathbb{R}^{N}\right) \\
\varepsilon \nabla u_{\varepsilon} \rightarrow 0 & \text { in } L^{2}\left(\mathbb{R}^{N}\right) \\
u_{\varepsilon} \rightarrow u & \text { in } L_{\text {loc }}^{2}\left(\mathbb{R}^{N}\right) \text { and a. e. in } \mathbb{R}^{N} .
\end{aligned}
$$

Moreover, from Proposition 5(ii) we know that

$$
\left\|u_{\varepsilon}\right\|_{L^{\infty}} \leq \frac{1}{\lambda}\|f\|_{\infty}
$$

We now consider the entropy inequality for the approximating solutions $u_{\varepsilon}$, i.e.,

$$
\begin{aligned}
& \int_{\mathbb{R}^{N}}\left(f(x)-\varepsilon u_{\varepsilon}(x)-b\left(u_{\varepsilon}(x)\right)\right) \eta^{\prime}\left(u_{\varepsilon}(x)\right) \varphi(x) \mathrm{d} x-\varepsilon \int_{\mathbb{R}^{N}} \nabla\left(\eta\left(u_{\varepsilon}(x)\right) \cdot \nabla \varphi(x) \mathrm{d} x\right. \\
& \quad+C(N, s) \int_{\mathbb{R}^{N}} \int_{\{|z| \geq r\}} \eta^{\prime}\left(u_{\varepsilon}(x)\right) \frac{u_{\varepsilon}(x+z)-u_{\varepsilon}(x)}{|z|^{N+s}} \varphi(x) \mathrm{d} z \mathrm{~d} x \\
& \quad+C(N, s) \int_{\mathbb{R}^{N}} \int_{\{|z| \leq r\}} \eta\left(u_{\varepsilon}(x)\right) \frac{\varphi(x+z)-\varphi(x)-\nabla \varphi(x) \cdot z}{|z|^{N+s}} \mathrm{~d} z \mathrm{~d} x \\
& \quad+\int_{\mathbb{R}^{N}} \phi_{\varepsilon}\left(u_{\varepsilon}(x)\right) \cdot \nabla \varphi(x) \mathrm{d} x \geq 0
\end{aligned}
$$

and it remains to prove that we can pass to the limit with $\varepsilon \rightarrow 0$ in (28) using (23)-(27). We would then obtain $u \in H^{\frac{s}{2}}\left(\mathbb{R}^{N}\right) \cap L^{\infty}\left(\mathbb{R}^{N}\right)$ which satisfies the entropy inequality and is therefore the desired entropy solution of problem $\left(\mathscr{P}_{f}\right)$ (Notice that $b(u) \in L^{1}\left(\mathbb{R}^{N}\right)$ follows from Proposition 5(i), (26) and Fatou's Lemma). 
With the above convergence results, we can calculate

$$
\begin{gathered}
\int_{\mathbb{R}^{N}}\left(f(x)-\varepsilon u_{\varepsilon}(x)-b\left(u_{\varepsilon}(x)\right)\right) \eta^{\prime}\left(u_{\varepsilon}(x)\right) \varphi(x) \mathrm{d} x \\
\rightarrow \int_{\mathbb{R}^{N}}(f(x)-b(u(x))) \eta^{\prime}(u(x)) \varphi(x) \mathrm{d} x
\end{gathered}
$$

for $\varepsilon \rightarrow 0$. Indeed, as $b$ and $\eta^{\prime}$ are continuous, by (26) it is clear that the integrand converges pointwise almost everywhere on $\mathbb{R}^{N}$ to $(f-b(u)) \eta^{\prime}(u) \varphi$. As $\left(u_{\varepsilon}\right)_{\varepsilon}$ is bounded in $L^{\infty}\left(\mathbb{R}^{N}\right), \varepsilon u_{\varepsilon} \rightarrow 0$ in $L^{2}\left(\mathbb{R}^{N}\right)$ and $\varphi$ has compact support, one can easily pass to the limit in the integral using the dominated convergence theorem of Lebesgue. Furthermore, we have $\varepsilon \nabla\left(\eta\left(u_{\varepsilon}(x)\right)\right) \cdot \nabla \varphi(x)=$ $\varepsilon \nabla u_{\varepsilon}(x) \eta^{\prime}\left(u_{\varepsilon}(x)\right) \cdot \nabla \varphi(x)$ and from this, we get for the second integral, together with (25), that

$$
\varepsilon \int_{\mathbb{R}^{N}} \nabla\left(\eta\left(u_{\varepsilon}(x)\right) \nabla \varphi(x) \mathrm{d} x \rightarrow 0\right.
$$

for $\varepsilon \rightarrow 0$. For the nonlocal terms we argue as follows: First we get

$$
\eta^{\prime}\left(u_{\varepsilon}(x)\right) \frac{u_{\varepsilon}(x+z)-u_{\varepsilon}(x)}{|z|^{N+s}} \varphi(x) \rightarrow \eta^{\prime}(u(x)) \frac{u(x+z)-u(x)}{|z|^{N+s}} \varphi(x)
$$

for almost all $x \in \mathbb{R}^{N}, z \in B_{r}(0)^{c}$ and $\varepsilon \rightarrow 0$. Further calculations show that

$$
\left|\eta^{\prime}\left(u_{\varepsilon}(x)\right) \frac{u_{\varepsilon}(x+z)-u_{\varepsilon}(x)}{|z|^{N+s}} \varphi(x)\right| \leq C \frac{|\varphi(x)|}{|z|^{N+s}} \in L^{1}\left(\mathbb{R}^{N} \times B_{r}(0)^{c}\right),
$$

for a constant $C>0$, not depending on $\varepsilon$, since $\left(u_{\varepsilon}\right)_{\varepsilon}$ is uniformly bounded in $L^{\infty}\left(\mathbb{R}^{N}\right)$. With Lebesgue's dominated convergence theorem, we then get

$$
\begin{aligned}
& C(N, s) \int_{\mathbb{R}^{N}} \int_{\{|z| \geq r\}} \eta^{\prime}\left(u_{\varepsilon}(x)\right) \frac{u_{\varepsilon}(x+z)-u_{\varepsilon}(x)}{|z|^{N+s}} \varphi(x) \mathrm{d} z \mathrm{~d} x \\
& \rightarrow C(N, s) \int_{\mathbb{R}^{N}} \int_{\{|z| \geq r\}} \eta^{\prime}(u(x)) \frac{u(x+z)-u(x)}{|z|^{N+s}} \varphi(x) \mathrm{d} z \mathrm{~d} x
\end{aligned}
$$

for $\varepsilon \rightarrow 0$. For the singular part

$$
\int_{\mathbb{R}^{N}} \int_{\{|z| \leq r\}} \eta\left(u_{\varepsilon}(x)\right) \frac{\varphi(x+z)-\varphi(x)-\nabla \varphi(x) \cdot z}{|z|^{N+s}} \mathrm{~d} z \mathrm{~d} x
$$

we also get that

$$
\eta\left(u_{\varepsilon}(x)\right) \frac{\varphi(x+z)-\varphi(x)-\nabla \varphi(x) \cdot z}{|z|^{N+s}} \rightarrow \eta(u(x)) \frac{\varphi(x+z)-\varphi(x)-\nabla \varphi(x) \cdot z}{|z|^{N+s}}
$$


for almost all $x \in \mathbb{R}^{N}, z \in B_{r}(0)$ and $\varepsilon \rightarrow 0$. We also notice that $\mid \varphi(x+$ $z)-\varphi(x)-\left.\nabla \varphi(x) \cdot z\left|\leq\left\|D^{2} \varphi\right\|_{L^{\infty}}\right| z\right|^{2}$ for all $x, z \in \mathbb{R}^{N}$ holds. Therefore, the integrand is dominated by the function

$$
C \frac{\left\|D^{2} \varphi\right\|_{L^{\infty}}}{|z|^{N+s-2}} \chi_{\{|z| \leq r\}} \chi_{\{|x| \leq R+r\}} \in L^{1}\left(\mathbb{R}^{N} \times B_{r}(0)\right),
$$

for a constant $C>0$, not depending on $\varepsilon$, where $R>0$ is such that $\operatorname{supp} \varphi \subseteq$ $B_{R}(0)$. Again, by Lebesgue's dominated convergence theorem, we get that

$$
\begin{aligned}
& \int_{\mathbb{R}^{N}} \int_{\{|z| \leq r\}} \eta\left(u_{\varepsilon}(x)\right) \frac{\varphi(x+z)-\varphi(x)-\nabla \varphi(x) \cdot z}{|z|^{N+s}} \mathrm{~d} z \mathrm{~d} x \\
& \quad \rightarrow \int_{\mathbb{R}^{N}} \int_{\{|z| \leq r\}} \eta(u(x)) \frac{\varphi(x+z)-\varphi(x)-\nabla \varphi(x) \cdot z}{|z|^{N+s}} \mathrm{~d} z \mathrm{~d} x
\end{aligned}
$$

for $\varepsilon \rightarrow 0$. Since, again, $\left(u_{\varepsilon}\right)_{\varepsilon}$ is uniformly bounded in $L^{\infty}\left(\mathbb{R}^{N}\right)$, there exists $\varepsilon_{0}>0$ such that $\left\|u_{\varepsilon}\right\|_{\infty} \leq \frac{1}{\varepsilon} \forall 0<\varepsilon<\varepsilon_{0}$. Therefore, we have $T_{1 / \varepsilon}\left(u_{\varepsilon}\right)=u_{\varepsilon}$ $\forall 0<\varepsilon<\varepsilon_{0}$ and in this case we get

$$
\int_{\mathbb{R}^{N}} \phi_{\varepsilon}\left(u_{\varepsilon}(x)\right) \nabla \varphi(x) \mathrm{d} x=\int_{\mathbb{R}^{N}} \phi\left(u_{\varepsilon}(x)\right) \nabla \varphi(x) \mathrm{d} x \rightarrow \int_{\mathbb{R}^{N}} \phi(u(x)) \nabla \varphi(x) \mathrm{d} x
$$

for $\varepsilon \rightarrow 0$. This follows by applying Lebesgue's dominated convergence theorem, since

$$
\phi\left(u_{\varepsilon}(x)\right)=\int_{0}^{u_{\varepsilon}(x)} \eta^{\prime}(\sigma) F^{\prime}(\sigma) \mathrm{d} \sigma \rightarrow \int_{0}^{u(x)} \eta^{\prime}(\sigma) F^{\prime}(\sigma) \mathrm{d} \sigma=\phi(u(x))
$$

almost everywhere in $\mathbb{R}^{N}$ for $\varepsilon \rightarrow 0$ from $(26), \phi\left(u_{\varepsilon}\right)$ is uniformly bounded in $L^{\infty}\left(\mathbb{R}^{N}\right)$ and the integral is taken over the compact support of $\varphi$.

This completes the proof of Theorem 1.

Author contributions All listed authors have contributed to the manuscript substantially and have agreed to the final submitted version.

Funding Open Access funding enabled and organized by Projekt DEAL.

\section{Declarations}

Conflict of interest The authors declare that they have no conflict of interest.

Open Access. This article is licensed under a Creative Commons Attribution 4.0 International License, which permits use, sharing, adaptation, distribution and reproduction in any medium or format, as long as you give appropriate credit to the original author(s) and the source, provide a link to the Creative Commons licence, and indicate if changes were made. The images or other third party material in 
this article are included in the article's Creative Commons licence, unless indicated otherwise in a credit line to the material. If material is not included in the article's Creative Commons licence and your intended use is not permitted by statutory regulation or exceeds the permitted use, you will need to obtain permission directly from the copyright holder. To view a copy of this licence, visit http://creativecommons. org/licenses/by/4.0/.

\section{Appendix}

Proof of Proposition 3. (i) Let $u \in C_{b}^{2}\left(\mathbb{R}^{N}\right)$ be such that for all $x \in \mathbb{R}^{N}$ we have

$$
b(u(x))+\operatorname{div}(F(u(x)))+(-\Delta)^{\frac{s}{2}} u(x)=f(x) .
$$

Since $\eta \in C^{2}(\mathbb{R})$ is convex, it holds

$$
\eta(b)-\eta(a) \geq \eta^{\prime}(a)(b-a)
$$

for all $a, b \in \mathbb{R}$. Let $a=u(x)$ and $b=u(x+z)$, for $x, z \in \mathbb{R}^{N}$, then it follows

$$
\begin{aligned}
& \eta(u(x+z))-\eta(u(x))-\nabla \eta(u(x)) \cdot z \\
& \quad \geq \eta^{\prime}(u(x))(u(x+z)-u(x)-\nabla u(x) \cdot z)
\end{aligned}
$$

and therefore

$$
\begin{aligned}
\eta^{\prime}(u(x))(-\Delta)^{\frac{s}{2}} u(x) & -C(N, s) \int_{\{|z| \geq r\}} \eta^{\prime}(u(x)) \frac{u(x+z)-u(x)}{|z|^{N+s}} \mathrm{~d} z \\
& -C(N, s) \int_{\{|z| \leq r\}} \eta^{\prime}(u(x)) \frac{u(x+z)-u(x)-\nabla u(x) \cdot z}{|z|^{N+s}} \mathrm{~d} z \\
\geq & -C(N, s) \int_{\{|z| \geq r\}} \eta^{\prime}(u(x)) \frac{u(x+z)-u(x)}{|z|^{N+s}} \mathrm{~d} z \\
& -C(N, s) \int_{\{|z| \leq r\}} \frac{\eta(u(x+z))-\eta(u(x))-\nabla \eta(u(x)) \cdot z}{|z|^{N+s}} \mathrm{~d} z .
\end{aligned}
$$

If we multiply equation (29) pointwise with $\eta^{\prime}(u(x))$, for all $x \in \mathbb{R}^{N}$, and use the inequality (30), we get

$$
\begin{aligned}
& 0 \geq(b(u(x))-f(x)) \eta^{\prime}(u(x))+\operatorname{div}(\phi(u(x))) \\
& -C(N, s) \int_{\{|z| \geq r\}} \eta^{\prime}(u(x)) \frac{u(x+z)-u(x)}{|z|^{N+s}} \mathrm{~d} z
\end{aligned}
$$




$$
-C(N, s) \int_{\{|z| \leq r\}} \frac{\eta(u(x+z))-\eta(u(x))-\nabla \eta(u(x)) \cdot z}{|z|^{N+s}} \mathrm{~d} z,
$$

where we considered the structure of the entropy flux $\phi^{\prime}=\eta^{\prime} F^{\prime}$ to get $\operatorname{div}(\phi(u(x)))=\operatorname{div}(F(u(x))) \eta^{\prime}(u(x))$. If we now multiply this inequality pointwise with $\varphi(x), \varphi \in \mathscr{D}\left(\mathbb{R}^{N}\right), \varphi \geq 0$, and then integrate with respect to $x$ over $\mathbb{R}^{N}$, we get, by applying the integration-by-parts rule to the divergence term, that

$$
\begin{aligned}
& \int_{\mathbb{R}^{N}}(f(x)-b(u(x))) \eta^{\prime}(u(x)) \varphi(x)+\phi(u(x)) \cdot \nabla \varphi(x) \mathrm{d} x \\
& \quad+C(N, s) \int_{\mathbb{R}^{N}} \int_{\{|z| \geq r\}} \eta^{\prime}(u(x)) \frac{u(x+z)-u(x)}{|z|^{N+s}} \varphi(x) \mathrm{d} z \mathrm{~d} x \\
& \quad+C(N, s) \int_{\mathbb{R}^{N}} \int_{\{|z| \leq r\}} \frac{\eta(u(x+z))-\eta(u(x))-\nabla \eta(u(x)) \cdot z}{|z|^{N+s}} \varphi(x) \mathrm{d} z \mathrm{~d} x \geq 0 .
\end{aligned}
$$

Now we have to transfer the fractional derivative in the last term of the left-hand side onto the test function $\varphi$. With Taylor's Formula and Fubini's Theorem we get

$$
\begin{aligned}
\int_{\mathbb{R}^{N}} & \int_{\{|z| \leq r\}} \frac{\eta(u(x+z))-\eta(u(x))-\nabla \eta(u(x)) \cdot z}{|z|^{N+s}} \varphi(x) \mathrm{d} z \mathrm{~d} x \\
= & \int_{0}^{1} \int_{\mathbb{R}^{N}} \int_{\{|z| \leq r\}} \frac{(1-\tau) \mathrm{D}^{2}(\eta(u(x+\tau z))) z \cdot z}{|z|^{N+s}} \varphi(x) \mathrm{d} z \mathrm{~d} x \mathrm{~d} \tau \\
= & \int_{0}^{1} \int_{\{|z| \leq r\}} \frac{(1-\tau)}{|z|^{N+s}}\left(\int_{\mathbb{R}^{N}} \mathrm{D}^{2}(\eta(u(x+\tau z))) z \cdot(\varphi(x) z) \mathrm{d} x\right) \mathrm{d} z \mathrm{~d} \tau \\
= & -\int_{0}^{1} \int_{\{|z| \leq r\}} \frac{(1-\tau)}{|z|^{N+s}}\left(\int_{\mathbb{R}^{N}} \nabla(\eta(u(x+\tau z))) \cdot z(\nabla \varphi(x) \cdot z) \mathrm{d} x\right) \mathrm{d} z \mathrm{~d} \tau \\
= & -\int_{0}^{1} \int_{\mathbb{R}^{N}} \int_{\{|z| \leq r\}} \frac{(1-\tau)}{|z|^{N+s}} \nabla(\eta(u(x))) \cdot z(\nabla \varphi(x+\tau z) \cdot z) \mathrm{d} z \mathrm{~d} x \mathrm{~d} \tau,
\end{aligned}
$$

where we used the substitution of variables $(\tau, x, z) \rightarrow(\tau, x+\tau z,-z)$. Interchanging the roles of $\eta(u)$ and $\varphi$ in the last equality and calculating the exact steps backwards, we then get

$$
\int_{\mathbb{R}^{N}} \int_{\{|z| \leq r\}} \frac{\eta(u(x+z))-\eta(u(x))-\nabla \eta(u)(x) \cdot z}{|z|^{N+s}} \varphi(x) \mathrm{d} z \mathrm{~d} x
$$




$$
=\int_{\mathbb{R}^{N}} \int_{\{|z| \leq r\}} \eta(u(x)) \frac{\varphi(x+z)-\varphi(x)-\nabla \varphi(x) \cdot z}{|z|^{N+s}} \mathrm{~d} z \mathrm{~d} x
$$

and hereby we get the claim.

(ii) Let $u$ be an entropy solution of $\left(\mathscr{P}_{f}\right)$. If we first choose $\eta(r)=r \forall r \in \mathbb{R}$, then it follows

$$
\begin{aligned}
& \int_{\mathbb{R}^{N}}(f(x)-b(u(x))) \varphi(x)+F(u(x)) \cdot \nabla \varphi(x) \mathrm{d} x \\
& \quad+C(N, s) \int_{\mathbb{R}^{N}} \int_{\{|z| \geq r\}} \frac{u(x+z)-u(x)}{|z|^{N+s}} \varphi(x) \mathrm{d} z \mathrm{~d} x \\
& \quad+C(N, s) \int_{\mathbb{R}^{N}} \int_{\{|z| \leq r\}} u(x) \frac{\varphi(x+z)-\varphi(x)-\nabla \varphi(x) \cdot z}{|z|^{N+s}} \mathrm{~d} z \mathrm{~d} x \geq 0
\end{aligned}
$$

for all $\varphi \in \mathscr{D}\left(\mathbb{R}^{N}\right), \varphi \geq 0$. Analogously, for $\eta(r)=-r \forall r \in \mathbb{R}$, we can show the converse inequality to then get equality. By substituion of variables $(x, z) \mapsto(x+z,-z)$ and Fubini's Theorem, we also get

$$
\begin{aligned}
& \int_{\mathbb{R}^{N}} \int_{\{|z| \geq r\}} \frac{u(x+z)-u(x)}{|z|^{N+s}} \varphi(x) \mathrm{d} z \mathrm{~d} x \\
& =\int_{\mathbb{R}^{N}} \int_{\{|z| \geq r\}} u(x) \frac{\varphi(x+z)-\varphi(x)}{|z|^{N+s}} \mathrm{~d} z \mathrm{~d} x .
\end{aligned}
$$

With the representation of the fractional Laplacian in (3) the claim follows.

Proof of Lemma 5. By the divergence theorem of Gauss it is well known that, on every bounded Lipschitz domain $\Omega \subseteq \mathbb{R}^{N}$, it holds

$$
\int_{\Omega} F_{\varepsilon}(u(x)) \nabla u(x) \mathrm{d} x=0
$$

for all $u \in H_{0}^{1}(\Omega)$. Let $\left(u_{n}\right)_{n} \subseteq C_{c}^{\infty}\left(\mathbb{R}^{N}\right)$ be such that supp $u_{n} \subseteq B_{n}(0)=: \Omega_{n}$ for every $n \in \mathbb{N}$ and

$$
u_{n} \rightarrow u \quad \text { in } H^{1}\left(\mathbb{R}^{N}\right) .
$$

Therefore, we get

$$
\int_{\mathbb{R}^{N}} F_{\varepsilon}(u(x)) \nabla u(x) \mathrm{d} x=\lim _{n \rightarrow \infty} \int_{\Omega_{n}} F_{\varepsilon}\left(u_{n}(x)\right) \nabla u_{n}(x) \mathrm{d} x=0 .
$$




\section{References}

[1] Alibaud, N.: Entropy formulation for fractal conservation laws. J. Evol. Equ. 7(1), 145-175 (2007)

[2] Alibaud, N., Andreianov, B.: Non-uniqueness of weak solutions for the fractal Burgers equation. Ann. Inst. H. Poincaré Anal. Non Linéaire 27(4), 997-1016 (2010)

[3] Alibaud, N., Andreianov, B., Bendahmane, M.: Renormalized solutions of the fractional Laplace equation. C. R. Math. Acad. Sci. Paris 348(13-14), 759-762 (2010)

[4] Bénilan, P., Crandall, M.G., Pazy, A.: Nonlinear evolution equations governed by accretive operators. Preprint, http://www.math.tu-dresden.de/ chill/files/

[5] Bénilan, P., Boccardo, L., Gallouët, Th., Gariepy, R., Pierre, M., Vázquez, J.L.: An $L^{1}$ theory of existence and uniqueness of solutions of nonlinear elliptic equations. Ann. Sc. Norm. Super. Pisa Cl. Sci. (4) 22(2), 241-273 (1995)

[6] Bhauryal, N., Koley, U., Vallet, G.: A fractional degenerate parabolic-hyperbolic Cauchy problem with noise. J. Differ. Equ. 284, 433-521 (2021)

[7] Bonforte, M., Vázquez, J.L.: Quantitative local and global a priori estimates for fractional nonlinear diffusion equations. Adv. Math. 250, 242-284 (2014)

[8] Cifani, S., Jakobsen, E.R., Karlsen, K.H.: The discontinuous Galerkin method for fractal conservation laws. IMA J. Numer. Anal. 31(3), 1090-1122 (2011)

[9] Demengel, F., Demengel, G.: Espaces fonctionnels. Utilisation dans la résolution des équations aux dérivées partielles. Savoirs Actuels (Les Ulis). EDP Sciences, Les Ulis; CNRS Éditions, Paris, 2007. xii+467 p. ISBN: 978-2-86883-996-1; 9782-271-06581-0

[10] Di Nezza, E., Palatucci, G., Valdinoci, E.: Hitchhiker's guide to the fractional Sobolev spaces. Bull. Sci. Math. 136(5), 521-573 (2012)

[11] Droniou, J., Gallouët, T., Vovelle, J.: Global solution and smoothing effect for a non-local regularization of a hyperbolic equation. Dedicated to Philippe Bénilan. J. Evol. Equ. 3(3), 499-521 (2003)

[12] Droniou, J., Imbert, C.: Fractal first-order partial differential equations. Arch. Ration. Mech. Anal. 182(2), 299-331 (2006)

[13] Emmrich, E.: Gewöhnliche und Operator-Differentialgleichungen: Eine integrierte Einführung in Randwertprobleme und Evolutionsgleichungen für Studierende. Vieweg, Wiesbaden (2004)

[14] Evans, L.C.: Partial Differential Equations, 2nd edn. Graduate Studies in Mathematics, 19. American Mathematical Society, Providence, RI, 2010. xxii+749 pp. ISBN: 978-0-8218-4974-3

[15] Kruzhkov, S.N.: First order quasilinear equations with several independent variables. Math. Sb. (N.S.) 81(123), 228-255 (1970)

[16] Spener, A., Weber, F., Zacher, R.: The fractional Laplacian has infinite dimension. Commun. Partial Differ. Equ. 45(1), 57-75 (2020)

[17] Zeidler, E.H.: Nonlinear Functional Analysis and Its Applications. I. Fixed-point theorems. Springer, New York (1986) 
Niklas Grossekemper, Petra Wittbold and Aleksandra Zimmermann

Faculty of Mathematics

University of Duisburg-Essen

Thea-Leymann-Str. 9

45127 Essen

Germany

e-mail: aleksandra.zimmermann@uni-due.de

Niklas Grossekemper

e-mail: niklas.grossekemper@uni-due.de

Petra Wittbold

email: petra.wittbold@uni-due.de

Received: January 8, 2021.

Accepted: August 14, 2021.

Publisher's Note Springer Nature remains neutral with regard to jurisdictional claims in published maps and institutional affiliations. 